\title{
Towards the understanding of the behavior of bio-based nanostructures during in vitro digestion Ana C Pinheiro ${ }^{1,2}$, Raquel FS Gonçalves ${ }^{1}$, Daniel A Madalena ${ }^{1}$ and António A Vicente ${ }^{1}$
}

The encapsulation of bioactive compounds in bio-based nanostructures is considered a hot topic in food technology, due to their huge potential in protecting the valuable bioactive compounds and providing new functionalities (e.g. increase of bioavailability). However, there are still some challenges that must be overcome before this technology can be entirely embraced by food industry, including the optimization of nanostructures' formulations to increase stability and bioactive compounds' bioavailability and the risk assessment of their use in food. Therefore, in recent years, efforts are being directed to the evaluation of the in vitro behavior of nanostructures during digestion/absorption. This evaluation can be challenging, however, there are opportunities to take advantage from the lessons learned from pharmaceutical industry and of the considerable progress in the development of more realistic in vitro models and in situ analysis techniques to more accurately predict the behavior of bio-based nanostructures once ingested.

\author{
Addresses \\ ${ }^{1}$ Centre of Biological Engineering, University of Minho, 4715-057 Braga, \\ Portugal \\ ${ }^{2}$ Instituto de Biologia Experimental e Tecnológica, Avenida da \\ República, Quinta-do-Marquês, Estação Agronómica Nacional, \\ Apartado 12, 2781-901 Oeiras, Portugal
}

Corresponding author: Vicente, António A (avicente@deb.uminho.pt)

\author{
Current Opinion in Food Science 2017, 15:79-86 \\ This review comes from a themed issue on Food physics and \\ material science \\ Edited by Rosiane Lopes da Cunha \\ For a complete overview see the Issue and the Editorial \\ Available online 6th July 2017 \\ http://dx.doi.org/10.1016/j.cofs.2017.06.005 \\ 2214-7993/C 2017 Elsevier Ltd. All rights reserved.
}

\section{Introduction}

The increase in consumers' awareness of the impact that food has on health has spurred the development of functional foods, that is, food containing bioactive compounds (e.g. carotenoids, vitamins, fatty acids, polyphenols, bioactive peptides, phytosterols, fibers and essential minerals) dispersed within their matrix. However, most bioactive compounds present low water solubility, poor bioavailability, sensitivity to deterioration during processing, shelf-life and digestion, and/or unpleasant sensory attributes, and therefore cannot be added directly into foods $\left[1^{\circ}\right]$. Delivery systems at nano-scale have attracted considerable interest worldwide over the past decade, due to their potential to enhance the bioactive compounds' bioavailability (i.e. amount of ingested bioactive compound that is absorbed and available for physiological functions) and stability, with minimal adverse sensory effects. However, alongside their great potential, nanostructures for food applications are also facing some concerns about possible toxicity. Only through the understanding of the digestive fate of nanostructures/ bioactive compounds it is possible to improve their performance and to conclude about nanostructures' safety. The present review highlights the latest (past 2-3 years) important advances in the development of bio-based delivery systems at nanoscale and in unraveling their behavior within the gastrointestinal (GI) tract, including an insight into the challenges associated with this in vitro evaluation and potential future directions (e.g. development and use of more sophisticated dynamic in vitro digestion models).

\section{Bio-based nanostructures for encapsulation of bioactive compounds}

By contrast to pharmaceutical industry, nanostructures for food applications must be entirely produced with food grade ingredients (e.g. lipids, proteins, polysaccharides, surfactants) and through approved processing operations (e.g. mixing, homogenizing thermal processing) [2]. Because of this reason, there is an increasing interest in founding new natural ingredients to produce bio-based nanostructures with good properties to incorporate in food products without interfering with their sensory characteristics.

There have been major advances in the design and production of bio-based nanostructures to encapsulate bioactive compounds for food applications. Till date, different bio-based nanostructures have been described in the available literature, including nanoemulsions, liposomes, nanostructured lipid carriers, polymeric nanoparticles, protein-polysaccharide complexes and nanohydrogels. These bio-based nanostructures present different sizes, structures, compositions and physicochemical properties and can offer numerous functionalities. Also, they typically present as principal advantages 
biodegradability, low toxicity, high loading capacity, improvement of oral absorption and controlled/sustainable release [3]. Also, bio-based nanostructures can be especially formulated to survive passage through different regions of the GI tract and then release the bioactive compounds at a specific location, thus maximizing their potential health benefits [4]. Some recent developments on nanoencapsulation of bioactive compounds for food applications, with special emphasis to their outcomes, can be found in Table 1 .
In the past years, there has been a tendency to design new bio-based nanostructures through the combination of existent nanomaterials (lipids and polymers), promoting a synergistic interaction between these nanomaterials. Furthermore, the development of new delivery systems, such as the combination of two (nano)structures (e.g. nanoparticles trapped within hydrogel beads [16]) could be a new approach for encapsulation of bioactive compounds with more stability, protection and efficient control release in food products.

\begin{tabular}{|c|c|c|c|c|}
\hline \multicolumn{5}{|c|}{ Recent advances in the development of bio-based nanostructures for encapsulation of bioactive compounds for food applications } \\
\hline Nano-delivery system & Bioactive compound & Nanostructure materials & Outcomes & Reference \\
\hline \multirow[t]{2}{*}{ Nanoemulsions } & $\begin{array}{l}\text { Astaxanthin } \\
\text { Lycopene }\end{array}$ & $\begin{array}{l}\text { Linseed oil } \\
\text { Tween } 20\end{array}$ & $\begin{array}{l}レ \text { Higher stability to storage and } \\
\text { environment conditions for emulsions with } \\
\text { size lower than } 200 \mathrm{~nm} \\
レ \text { Incorporation of combined antioxidants } \\
\text { increase the oxidative stability } \\
レ \text { Higher bioaccessibility of carotenoids } \\
\text { encapsulated in nanoemsulsions }\end{array}$ & [5] \\
\hline & Pterostilbene & $\begin{array}{l}\text { Olive oil } \\
\text { Flaxseed oil } \\
\text { Tween } 20\end{array}$ & $\begin{array}{l}\boldsymbol{V} \text { Higher percentage of FFA released for } \\
\text { olive oil } \\
\boldsymbol{V} \text { Similar bioaccessibility of pterostilbene } \\
\text { for olive oil and flaxseed oil nanoemulsions } \\
\boldsymbol{V} \text { Potential enhancement of the } \\
\text { permeability of the tight junctions for olive } \\
\text { oil nanoemulsions }\end{array}$ & [6] \\
\hline \multirow[t]{2}{*}{ Liposomes } & Fish oil & $\begin{array}{l}\text { Soy lecithin } \\
\text { Sunflower oil }\end{array}$ & $\begin{array}{l}\boldsymbol{V} \text { Encapsulation efficiency } \approx 92 \% \\
\boldsymbol{V} \text { Good stability } \\
\boldsymbol{V} \text { Incorporation in yogurt did not affect its } \\
\text { sensory quality and protected the fish oil }\end{array}$ & [7] \\
\hline & $\begin{array}{l}\text { Catechin } \\
\text { Epigallocatechin } \\
\text { gallate (EGCG) }\end{array}$ & Soy lecithin & $\begin{array}{l}\checkmark \text { High retention of bioactive compounds } \\
\text { in the cheese structure } \\
\checkmark \text { Increase of the antioxidant activity and } \\
\text { total phenolic content without affecting the } \\
\text { cheese characteristics } \\
\swarrow \text { Good protection of bioactive } \\
\text { compounds }\end{array}$ & [8] \\
\hline \multirow[t]{2}{*}{$\begin{array}{l}\text { Nanostructured lipid } \\
\text { carriers }\end{array}$} & Terpene & $\begin{array}{l}\text { Beeswax } \\
\text { MCT } \\
\text { Alkylpolyglucoside }\end{array}$ & $\begin{array}{l}\vee \text { Good stability } \\
\downarrow \text { High loading capacity } \\
\downarrow \text { Biphasic release: burst release and } \\
\text { sustained release } \\
\swarrow \text { Profile release dependent on chemical } \\
\text { structure of terpenes }\end{array}$ & [9] \\
\hline & Vitamin D3 & $\begin{array}{l}\text { Oleic acid } \\
\text { Glycerol monostearate } \\
\text { Tween } 80\end{array}$ & $\begin{array}{l} \\
\text { not affected by differences in } \mathrm{pH} \\
\\
\text { instable in alkaline conditions } \\
\\
\checkmark \text { Good storage stability } \\
レ \text { Capability to control release }\end{array}$ & {$[10]$} \\
\hline \multirow[t]{2}{*}{ Polymeric nanoparticles } & Curcumin & $\begin{array}{l}\text { Chitosan } \\
\text { Gum arabic } \\
\text { Tween } 80 \\
\text { Egg yolk phospholipid }\end{array}$ & $\begin{array}{l}\checkmark \text { High encapsulation efficiency and } \\
\text { loading capacity } \\
レ \text { Good physical stability } \\
\checkmark \text { Improvement of antioxidant activity } \\
\checkmark \text { Enhancement of delayed release in } \\
\text { Gl tract }\end{array}$ & {$[11]$} \\
\hline & $\mathrm{CoQ}_{10}$ & $\begin{array}{l}\text { Octenyl succinic anhydride } \\
\text { modified starch (OSA-ST) } \\
\text { Rice bran oil }\end{array}$ & $\begin{array}{l}レ \text { Good thermal and pH stability } \\
レ \text { Encapsulation efficiency } \approx 98.2 \% \\
\checkmark \text { Suitable for use in beverages, fruit } \\
\text { juices and baked goods }\end{array}$ & [12] \\
\hline
\end{tabular}




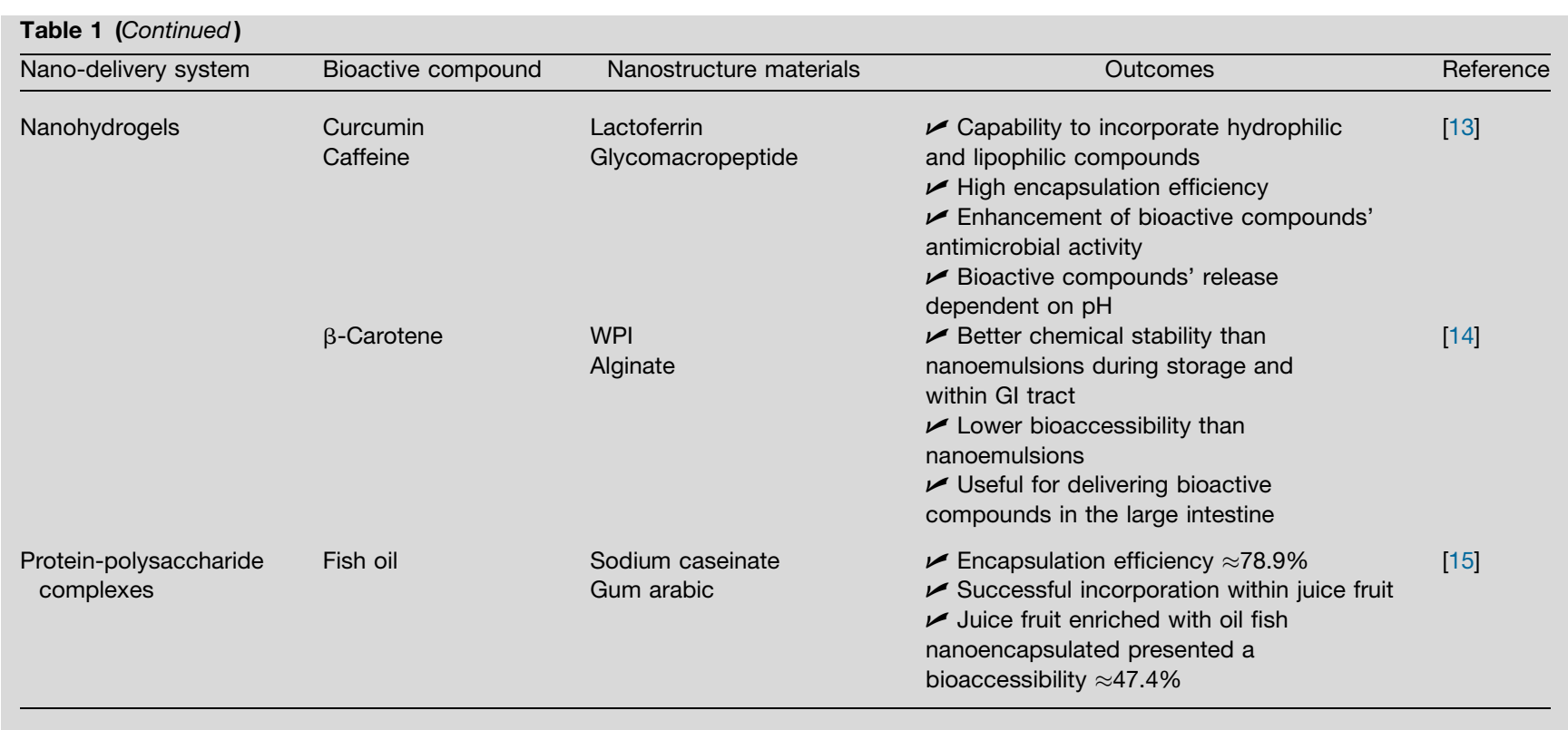

The selection of the most appropriate bio-based nanostructure for a particular application requires an understanding of the bioactive compound properties and of the nature of the food matrix in which it will be incorporated, as well as on their behavior within the GI tract.

\section{Gastrointestinal fate of bio-based nanostructures/bioactive compounds}

Bio-based nanostructures undergo a series of complex physicochemical and physiological processes as they pass through the different regions of the GI tract, before the release of the bioactive compound (Figure 1). Some of the key processes are (i) consecutive changes in the $\mathrm{pH}$ of the medium, which could alter nanostructures' electrical charge, and consequently their composition, structure and interactions; (ii) variations in the type and concentration of ions, which may impact the electrostatic interactions in the nanostructure through electrostatic screening or binding effects; (iii) presence of surface-active components (e.g. phospholipids and bile salts), which could lead to changes in nanostructures' interfacial composition; (iv) presence of enzymes able to digest components of the bio-based nanostructure, such as lipids (lipases), phospholipids (phospholipases), proteins (proteases), and starch (amylases); (v) temperature, which may cause changes in the physical state, molecular conformation or interactions of specific components, impacting nanostructures' digestibility; and (vi) flow/force profile which, besides mixing the various components, could lead to the breaking down of the delivery system's structure.

Different strategies have been used to control nanostructures' fate in the GI tract. For example, enzyme accessibility to a nanostructure may be controlled by creating physical barriers between the nanostructure and the surrounding aqueous phase containing the digestive enzymes. It has been demonstrated that emulsions' behavior during digestion can be modulated by coating them with biopolymers [17], nanostructures [18] or conjugates [19].

Thus, the biological fate of bio-based nanostructures will be dependent not only on their initial physicochemical characteristics (e.g. composition, size, structure, interfacial properties and physical state), but also on the extension of the changes experienced as they pass within the GI tract. It is know that at nanoscale, the biological fate of the delivery systems and bioactive compounds incorporated within may be altered, influencing their absorption, distribution, metabolism and excretion, and consequently their potential toxicity [20]. However, in contrast to inorganic nanoparticles (e.g. gold, silver), the absorption of intact bio-based nanostructures into the systemic circulation is unlikely, due the changes after undergone gastric and intestinal digestion, being their accumulation in the organs, and consequent toxic effect, also improbable. In any case, the transformation and retention of biobased nanostructures are related to their physicochemical properties, and therefore, the evaluation of their safety must be done in case-by-case basis.

Regarding the bioactive compounds, different mechanisms can be involved in their release from nanostructures (e.g. diffusion, swelling, erosion, fragmentation, dissolution, stimuli response) and, depending on the system and environmental conditions, a different mechanism may prevail [21]. Two main transport mechanisms can be involved in their passage across the intestinal epithelium: (1) between cells via tight junctions - paracellular route or (2) through intestinal membrane cells - transcellular route $\left[22^{\circ}\right]$. The bioactive compounds can undergo chemical degradation under harsh GI conditions or may be 


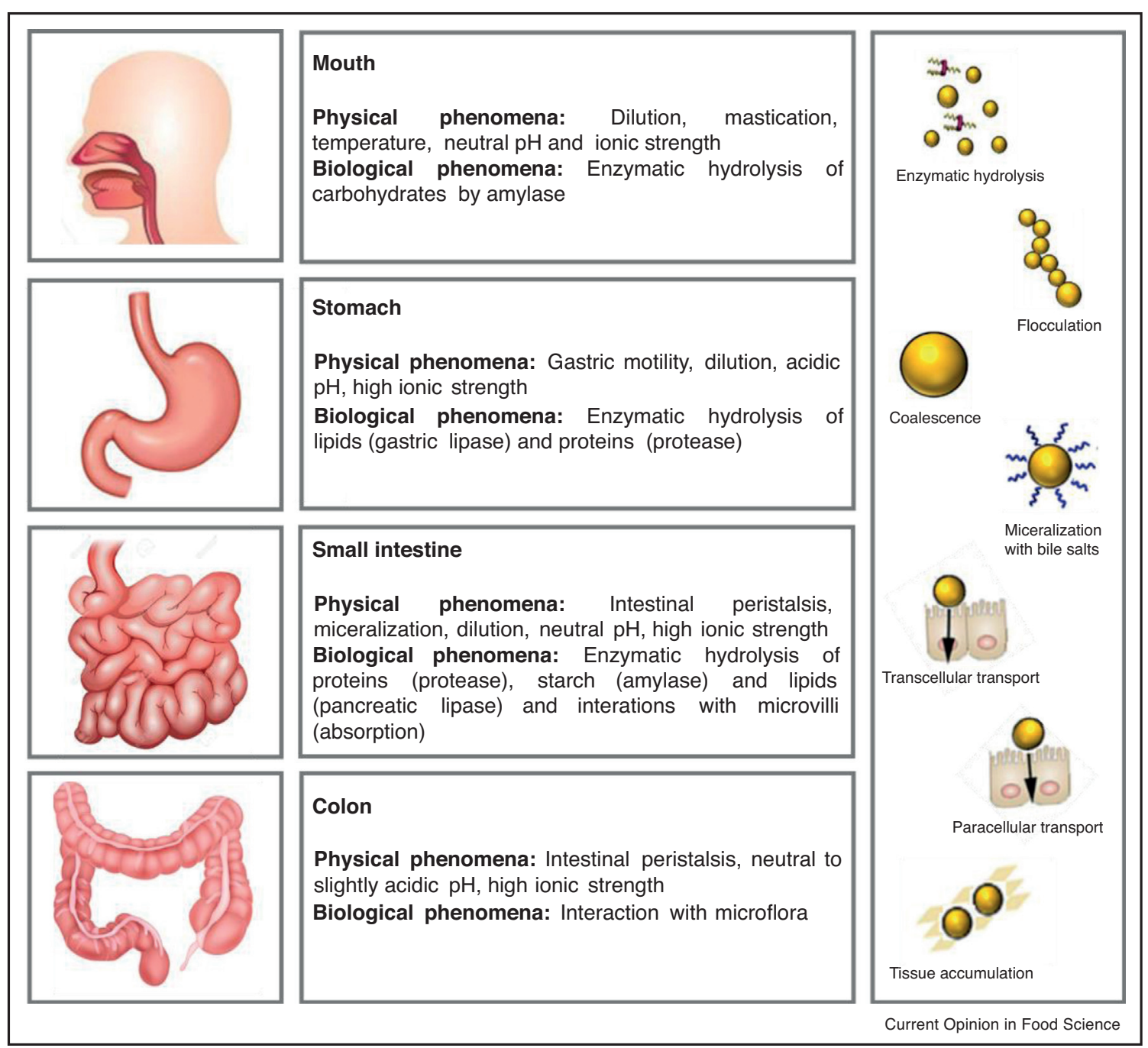

Physiological and physicochemical changes that bio-based nanostructures can undergo as they pass through the Gl tract.

metabolized by different enzymes during their passage from the GIT tract, to the epithelium cells, and then into the systemic circulation, leading to low bioavailability.

Understanding of the GI fate of bio-based nanostructures is therefore required to predict and increase their functionality (i.e. bioavailability of the bioactives compound) and to evaluate their potential toxicity.

\section{Strategies for the enhancement of the bioactive compounds' bioavailability}

Bioavailability is known to be dependent on the bioactive compounds' bioaccessibility, intestinal absorption and transformation in the GI tract [23]. The improvement of the bioavailability of a bioactive compound is essential to maintain their bioefficiency, that is, their health benefits. Several approaches have been used for the improvement of bioavailability of food bioactive compounds, most of them translated from pharmaceutical drug research. However, in order to establish a successful strategy to enhance the bioavailability of the bioactive compounds it is important to know the main factors limiting their bioavailability.

The use of nanoscale delivery systems can be per se a strategy to increase the bioavailability of different bioactive compounds, once they could facilitate the entering of the bioactive compounds through biological barriers, as well as avoiding their metabolic modifications. In fact, nanostructures such as nanoemulsions or solid lipid nanoparticles have been shown to increase the bioavailability of different lipophilic bioactive compounds [24,25].

Also, there is a high potential to further increase the bioactive compounds' bioavailability through the improvement of their intestinal permeability, which can 
be achieved by the incorporation of absorption enhancers in the delivery systems' formulation. Different foodgrade compounds, including bile salts, surfactants, fatty acids, polymers and herbal bioactives have shown the ability to improve the intestinal absorption of bioactive compounds [26]. The mode of action of absorption enhancers can be either paracellular, by the opening of tight junctions, or transcellular by increasing the plasma membrane permeability, or a combination of both [27]. Other potential approach to improve the bioavailability of bioactive compounds is the inhibition of intestinal cell transporters (e.g. ATP binding cassette (ABC) transport proteins) once they can be involved in the efflux of bioactive compounds back into the intestinal lumen, reducing bioavailability [28].

Moreover, the co-ingestion of bioactive compounds with excipient foods is also a bioavailability enhancement strategy that has gained much attention in the recent years. In this case, the bioactive compound (that might be left in its natural source) is consumed with an excipient food specially formulated to control bioactive compounds' release, solubilization, transport, metabolism and absorption within the GI tract, boosting their bioavailability [29]. In fact, it has been suggested that delivery systems containing mixed colloidal particles (protein and lipid nanoparticles) may be designed to increase the chemical stability and bioaccessibility of lipophilic bioactive compounds [30].

\section{Challenges in tracking bio-based nanostructures digestion}

There are still some challenges that must be overcome regarding the evaluation of bio-based nanostructures during digestion. For instance, digestion secretions (i.e. presence of enzymes, salts and bile salts), sample dilution (during digestion) and sample preparation (e.g. centrifugation) may influence the overall results obtained from the in vitro digestion (e.g. bioavailability determination or bio-based nanostructure tracking).

Centrifugation [31,32,33] and sample dilution [33] are often used as a pre-treatment, prior to sample analysis regarding spectrometry (i.e. spectrophotometry, spectrofluorimetry), chromatography (i.e. HPLC) and structural (i.e. DLS) analysis, respectively. Digestion samples may also be submitted to oxygen and light exposure, and temperature and $\mathrm{pH}$ variations, prior to analysis. For instance, in vitro digestion protocols often include an enzymatic deactivation step that may alter the structure of the assessed bio-based nanostructures or the bioactive compounds' activity. In fact, it is a common practice to submit digestion samples to low temperatures (i.e., through ice or liquid nitrogen) to slow the enzymatic digestion rates. Samples are also often submitted to high temperatures (i.e. $100{ }^{\circ} \mathrm{C}$ ) to stop enzymes' activity.
Moreover, due to the complexity of the mixture after digestion, a separation and purification step are often required. Several methods can be used for separation and purification of bio-based nanostructures after digestion simulation, including asymmetrical flow field flow fractionation [34]. It is expected that in the next few years, new reliable methods for the detection and characterization of nanostructures under different conditions are established, improving the traceability of nanostructures within the human body.

Despite the fact that these procedures are in fact crucial to determine bioactive compounds' bioavailability and nanostructures behavior under digestion, they can also be considered as a bottleneck that needs to be addressed to minimize misconceptions and false results. In situ analysis can solve some of the challenges discussed above since it does not require sample pre-treatment procedures and gives more reliable information, in real time, regarding nanostructural changes during digestion so that correlations can be made toward bioactive compounds' bioavailability. Recently, a 'real-time quantitative method' to track the structural changes that occur in emulsion interface, during in vitro intestinal conditions has been developed and these changes were correlated with the release of free fatty acids. The authors used fluorescent resonant energy transfer (FRET) to evaluate, in situ, the emulsion digestion under simulated intestinal conditions [35].

\section{Recent advances in the development of in vitro Gl systems}

In vitro GI models are currently used as the main tool to understand the behavior of bio-based nanostructures under GI conditions, minimizing the negative implications associated with in vivo studies (i.e., ethical, economical and technical constrains). However, these models are usually simple in vitro static models due to their lower cost and simplicity regarding protocol execution [36,37]. Only recently, dynamic in vitro GI models are being used to evaluate the behavior of bio-based nanostructures during digestion [38,39]. There is an ongoing awareness to developed more realistic in vitro GI models to study, not only food digestion, but potentially the behavior of nanostructures, reproducing the phenomena that occur during human digestion (e.g. gastric peristaltic movements) [40,41].

Recently an 'in vitro mechanical gastric system' (IMGS Figure 2a) using 3D printing technology has been developed. The authors used natural liquid latex and the model was coupled with four pairs of acrylic pistons, arranged on each side of $3 \mathrm{D}$ printed stomach to reproduce three contractions per minute. This system enables the study of important phenomena (i.e., propulsion, grinding and retropulsion) that occur during gastric digestion [40]. Other authors developed an 'in vitro gastric device' 
Figure 2

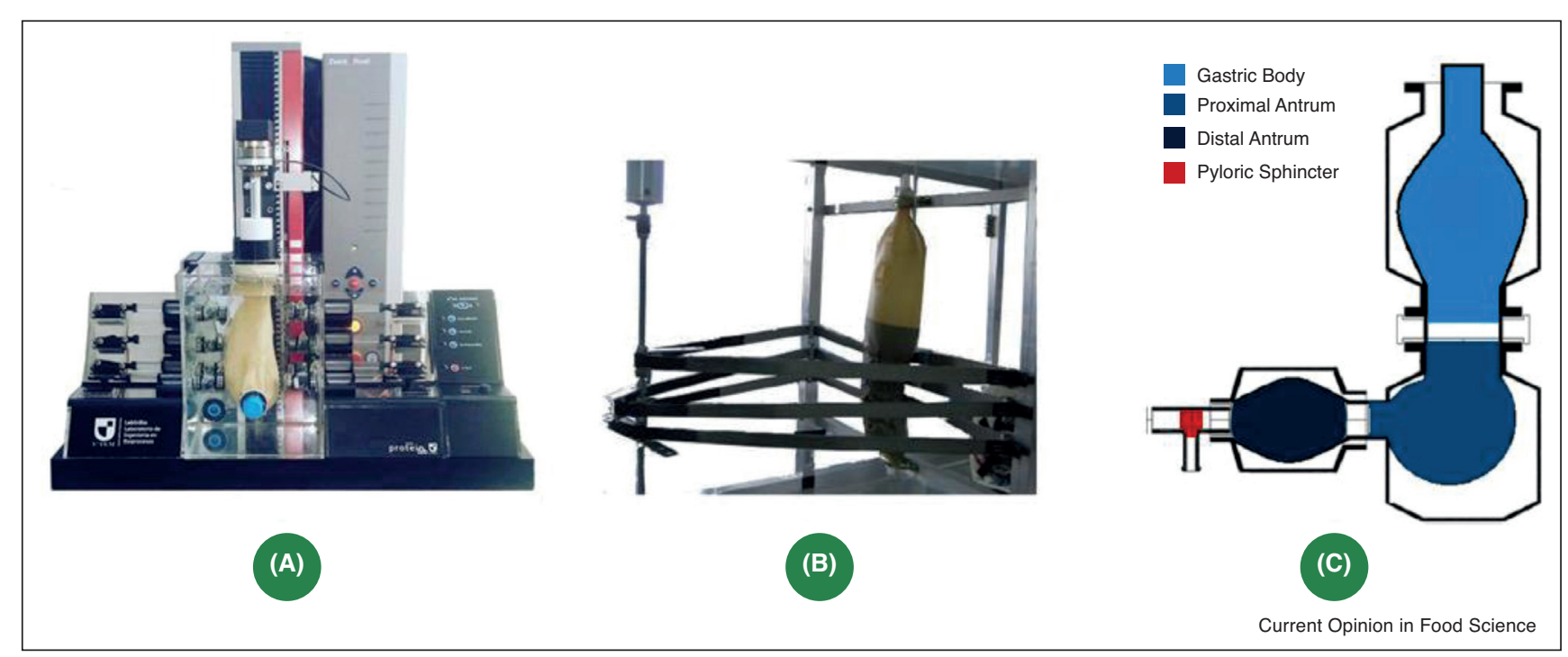

In vitro gastric models containing different mechanisms to simulate the human gastric peristaltic movements where (a) represents the IMGS, (b) represents the in vitro gastric device and (c) represents the TIMagc (reprinted from References $\left[40^{\circ}, 42,41\right]$, respectively, Copyright 2017 , with permission from Elsevier (a and c) and Dissolution Technologies, Inc. (b)).

coupled with a peristaltic mechanical system consisting in a three belt apparatus (Figure 2b). The authors used latex to achieve the appropriate deformation characteristics of the human stomach, with an oval shape. The belts were strategically positioned at the top, middle and bottom of the stomach model, reproducing three contractions per minute [42]. Also, an 'advanced gastric compartment' (TIMagc - Figure 2c) has been developed to study food gastric digestion. The authors built three compartments simulating the gastric body, proximal and distal antrum and the model was coupled with a valve to mimic the pyloric sphincter. The stomach peristalsis was simulated by synchronously contracting inner membranes on each compartment, simulating the human stomach motility [41].

The development of novel gastric systems that mimic both the anatomy and peristaltic gastric movements that occur during in vivo digestion is showing to be a current trend. These models use extensive in vivo data to recreate the conditions of the human stomach regarding $\mathrm{pH}$ variation during bolus digestion, stomach motility and secretion composition.

Recent advances were also observed regarding cellular studies, as a means to study bioactive compounds permeability in the small intestine. A tissue-engineered model has been established, by co-culturing Caco-2 absorptive cells, HT29-MTX mucus-producing cells and Raji B lymphocytes and the presence of M-cells (derived from stem cells) has been accessed [43]. The authors concluded that the presence of M-cells improved the bioactive compound transport efficiency, showing to be a more complete and reliable tool to perform permeability tests.

Despite the recent advantages in the field, there are still some bottlenecks that need to be addressed regarding the evaluation of bio-based nanostructures behavior under digestion. For instance, there is still a lack of agreement regarding the standardization of in vitro digestion protocols (i.e., both static and dynamic), despite the efforts of the COST action INFOGEST [34], so that inter-laboratory results can be compared and correlations can be established.

\section{Conclusions and future trends}

This review focus on the recent advances on the development of bio-based nanostructures for encapsulation of bioactive compounds, with special emphasis to their behavior under GI digestion. Efforts are being directed to the development of the next-generation delivery systems, with maximum functionality. Some examples can include mixed nanoparticle systems, nanoclusters, Trojan-horse nanoparticles and environmentally responsive nanoparticles.

However, there is still a lack of knowledge on the behavior of the nanostructures/bioactive compounds encapsulated in the GI tract, in particular on their potential adverse effects on human health. In order to respond to this concern, more realistic in vitro digestive/absorption models and more advanced analytical methods need to be developed, in order to understand the fate of nanostruc- 
tures within the GI tract, allowing the design of nanostructures with increased functionality and predicting the consequences of their intake. Even when using simple in vitro models, there is an evident need to develop standardized methods (e.g. for measure the bioavailability of bioactive compounds) that could allow a more accurate comparison of results between different laboratories. Finally, but not less important, a clear legislation and guidelines concerning their impact on human health and on environment must be assured. Although the regulatory framework on the use of nanostructures in foods is still in flux, it is expected an increase of control and regulation to assure the proper development and utilization of biobased nanostructures in foods.

\section{Conflict of interest}

The authors declare that there is no conflict of interest.

\section{Acknowledgements}

Ana C. Pinheiro acknowledge the Foundation for Science and Technology (FCT) for her fellowship (SFRH/BPD/101181/2014). This work was supported by Portuguese Foundation for Science and Technology (FCT) under the scope of the Project PTDC/AGR-TEC/5215/2014 and of the strategic funding of UID/BIO/04469/2013 unit, and COMPETE 2020 (POCI-01-0145-FEDER-006684) and BioTecNorte operation (NORTE01-0145-FEDER-000004) funded by the European Regional Development Fund under the scope of Norte2020 - Programa Operacional Regional do Norte. The authors would also like to thank the investment project $\mathrm{n}$ 017931, co-funded by Fundo Europeu de Desenvolvimento Regional (FEDER) through Programa Operacional Competitividade e Internacionalização (COMPETE 2020).

\section{References and recommended reading}

Papers of particular interest, published within the period of review, have been highlighted as:

- of special interest

$\bullet$ of outstanding interest

1. Livney YD: Nanostructured delivery systems in food: latest - developments and potential future directions. Curr Opin Food Sci 2015, 3:125-135.

This review presents the latest developments in nanodelivery systems for encapsulation of bioactive compounds, describing their types, structures, properties and production methods.

2. McClements DJ: Requirements for food ingredient and nutraceutical delivery systems. In Encapsulation Technologies and Delivery Systems for Food Ingredients and Nutraceuticals. Edited by Garti N, McClements DJ. Woodhead Publishing; 2012:318.

3. Shin GH, Kim JT, Park HJ: Recent developments in nanoformulations of lipophilic functional foods. Trends Food Sci Technol 2015, 46:144-157.

4. Jafari SM, McClements DJ: Nanotechnology approaches for increasing nutrient bioavailability. In Advances in Food and Nutrition Research, vol 81. Edited by Toldra F. Elsevier; 2017.

5. Sotomayor-Gerding D, Oomah BD, Acevedo F, Morales E, Bustamante M, Shene C, Rubilar M: High carotenoid bioaccessibility through linseed oil nanoemulsions with enhanced physical and oxidative stability. Food Chem 2016, 199:463-470.

6. Sun Y, Xia Z, Zheng J, Qiu P, Zhang L, McClements DJ, Xiao H Nanoemulsion-based delivery systems for nutraceuticals: influence of carrier oil type on bioavailability of pterostilbene. $J$ Funct Foods 2015, 13:61-70.
7. Ghorbanzade T, Jafari SM, Akhavan S, Hadavi R: Nanoencapsulation of fish oil in nano-liposomes and its application in fortification of yogurt. Food Chem 2017, 216:146-152.

8. Rashidinejad A, Birch EJ, Sun-Waterhouse D, Everett DW: Effect of liposomal encapsulation on the recovery and antioxidant properties of green tea catechins incorporated into a hard low-fat cheese following in vitro simulated gastrointestinal digestion. Food Bioprod Process 2016, 100(Part A):238-245.

9. Lasoń E, Sikora E, Ogonowski J, Tabaszewska M, Skoczylas Ł: Release study of selected terpenes from nanostructured lipid carriers. Colloids Surf A Physicochem Eng Asp 2016, 510:87-92.

10. Park SJ, Garcia CV, Shin GH, Kim JT: Development of nanostructured lipid carriers for the encapsulation and controlled release of vitamin D3. Food Chem 2017, 225:213219.

11. Tan C, Xie J, Zhang X, Cai J, Xia S: Polysaccharide-based nanoparticles by chitosan and gum arabic polyelectrolyte complexation as carriers for curcumin. Food Hydrocoll 2016, 57:236-245.

12. Cheuk SY, Shih FF, Champagne ET, Daigle KW, Patindol JA, Mattison CP, Boue SM: Nano-encapsulation of coenzyme Q10 using octenyl succinic anhydride modified starch. Food Chem 2015, 174:585-590.

13. Bourbon Al, Cerqueira MA, Vicente AA: Encapsulation and controlled release of bioactive compounds in lactoferringlycomacropeptide nanohydrogels: curcumin and caffeine as model compounds. J Food Eng 2016, 180:110-119.

14. Zhang Z, Zhang R, McClements DJ: Encapsulation of $\beta$ carotene in alginate-based hydrogel beads: impact on physicochemical stability and bioaccessibility. Food Hydrocoll 2016, 61:1-10.

15. Ilyasoglu H, El SN: Nanoencapsulation of EPA/DHA with sodium caseinate-gum arabic complex and its usage in the enrichment of fruit juice. LWT - Food Sci Technol 2014, 56:461468.

16. McClements DJ: The future of food colloids: next-generation nanoparticle delivery systems. Curr Opin Colloid Interface Sci 2017, 28:7-14.

17. Pinheiro $A C$, Coimbra MA, Vicente $A A$ : In vitro behaviour of curcumin nanoemulsions stabilized by biopolymer emulsifiers - effect of interfacial composition. Food Hydrocoll 2016.

18. Meshulam D, Lesmes U: Responsiveness of emulsions stabilized by lactoferrin nano-particles to simulated intestinal conditions. Food Funct 2014, 5:65-73.

19. Liu F, Ma C, Zhang R, Gao Y, Julian McClements D: Controlling the potential gastrointestinal fate of $\beta$-carotene emulsions using interfacial engineering: impact of coating lipid droplets with polyphenol-protein-carbohydrate conjugate. Food Chem 2017, 221:395-403.

20. McClements DJ: Edible lipid nanoparticles: digestion, absorption, and potential toxicity. Prog Lipid Res 2013, 52:409423.

21. Pinheiro AC, Bourbon Al, Cerqueira MA, Maricato É, Nunes C, Coimbra MA, Vicente AA: Chitosan/fucoidan multilayer nanocapsules as a vehicle for controlled release of bioactive compounds. Carbohydr Polym 2015, 115:1-9.

22. Martins J, Ramos Ó, Pinheiro A, Bourbon A, Silva H, Rivera M,

- Cerqueira M, Pastrana L, Malcata FX, González-Fernández Á et al.: Edible bio-based nanostructures: delivery, absorption and potential toxicity. Food Eng Rev 2015:1-23.

This review provides an insight into the innovations and challenges in the field of bio-based nanostructures for delivery of bioactive compounds via Gl tract.

23. McClements DJ, Li F, Xiao H: The nutraceutical bioavailability classification scheme: classifying nutraceuticals according to factors limiting their oral bioavailability. Ann Rev Food Sci Technol 2015, 6:299-327. 
24. Pandita D, Kumar S, Poonia N, Lather V: Solid lipid nanoparticles enhance oral bioavailability of resveratrol, a natural polyphenol. Food Res Int 2014, 62:1165-1174.

25. Parthasarathi S, Muthukumar SP, Anandharamakrishnan C: The influence of droplet size on the stability, in vivo digestion, and oral bioavailability of vitamin E emulsions. Food Funct 2016, 7:2294-2302.

26. Kesarwani K, Gupta R: Bioavailability enhancers of herbal origin: an overview. Asian Pac J Trop Biomed 2013, 3:253-266.

27. Maher S, Mrsny RJ, Brayden DJ: Intestinal permeation enhancers for oral peptide delivery. Adv Drug Deliv Rev 2016, 105(Part B).

28. Weinheimer M, Fricker G, Burhenne J, Mylius P, Schubert R: The application of P-gp inhibiting phospholipids as novel oral bioavailability enhancers - an in vitro and in vivo comparison. Eur J Pharm Sci 2016.

29. Li Q, Li T, Liu C, Dai T, Zhang R, Zhang Z, McClemnets DJ: Enhancement of carotenoid bioaccessibility from tomatoes using excipient emulsions: influence of particle size. Food Biophys 2017, 12:172-185.

30. Zou L, Zheng B, Zhang R, Zhang Z, Liu W, Liu C, Xiao H, McClements DJ: Enhancing the bioaccessibility of hydrophobic bioactive agents using mixed colloidal dispersions: curcumin-loaded zein nanoparticles plus digestible lipid nanoparticles. Food Res Int 2016, 81:74-82.

31. Liu F, Ma C, Zhang R, Gao Y, Julian McClements D: Controlling the potential gastrointestinal fate of beta-carotene emulsions using interfacial engineering: impact of coating lipid droplets with polyphenol-protein-carbohydrate conjugate. Food Chem 2017, 221:395-403.

32. Jayathunge KG, Stratakos AC, Cregenzan-Albertia O, Grant IR, Lyng J, Koidis A: Enhancing the lycopene in vitro bioaccessibility of tomato juice synergistically applying thermal and non-thermal processing technologies. Food Chem 2017, 221:698-705.

33. Madalena DA, Ramos ÓL, Pereira RN, Bourbon Al, Pinheiro AC, Malcata FX, Teixeira JA, Vicente AA: In vitro digestion and stability assessment of $\beta$-lactoglobulin/riboflavin nanostructures. Food Hydrocoll 2016, 58:89-97.

34. Zattoni A, Roda B, Borghi F, Marassi V, Reschiglian P: Flow field flow fractionation for the analysis of nanoparticles used in drug delivery. J Pharm Biomed Anal 2014, 87:53-61.
35. Pan $\mathrm{Y}$, Nitin N: Real-time measurements to characterize

- dynamics of emulsion interface during simulated intestinal digestion. Colloids Surf B Biointerfaces 2016, 141:233-241.

This paper reports the development of an in situ quantitative method using fluorescence resonant energy transfer, to study the structural changes that occur on emulsion interface during in vitro digestion and correlate them with the release of free fatty acids.

36. Martinez MJ, Martos G, Molina E, Pilosof AM: Reduced betalactoglobulin IgE binding upon in vitro digestion as a result of the interaction of the protein with casein glycomacropeptide. Food Chem 2016, 192:943-949.

37. Madureira AR, Pereira A, Pintado $\mathrm{M}$ : Chitosan nanoparticles loaded with 2,5-dihydroxybenzoic acid and protocatechuic acid: properties and digestion. J Food Eng 2016, 174:8-14.

38. Bourbon Al, Pinheiro AC, Cerqueira MA, Vicente AA: Influence of chitosan coating on protein-based nanohydrogels properties and in vitro gastric digestibility. Food Hydrocoll 2016, 60:109118.

39. Pinheiro $A C$, Coimbra MA, Vicente $A A$ : In vitro behaviour of curcumin nanoemulsions stabilized by biopolymer emulsifiers - effect of interfacial composition. Food Hydrocoll 2016, 52:460-467.

40. Barros L, Retamal C, Torres H, Zúñiga RN, Troncoso E:

- Development of an in vitro mechanical gastric system (IMGS) with realistic peristalsis to assess lipid digestibility. Food Res Int 2016, 90:216-225.

This paper introduces for the first time the 3D printing technology in the development of an in vitro gastric model. The designed model was also coupled with a mechanical system to reproduce the human gastric peristaltic movements.

41. Bellmann S, Lelieveld J, Gorissen T, Minekus M, Havenaar R: Development of an advanced in vitro model of the stomach and its evaluation versus human gastric physiology. Food Res Int 2016, 88(Part B):191-198.

42. Cascone S, Dalmoro A, Lamberti G, Titomanlio G, d'Amore M, Barba AA: In vitro simulation of human digestion: chemical and mechanical behavior. Dissolut Technol 2016, 23:16-23.

43. Araújo F, Pereira C, Costa J, Barrias C, Granja PL, Sarmento B: In vitro $\mathrm{M}$-like cells genesis through a tissue-engineered tripleculture intestinal model. J Biomed Mater Res Part B Appl Biomater 2016, 104:782-788. 